\title{
Changes in music tempo entrain movement related brain activity
}

\author{
Ian Daly ${ }^{1}$, James Hallowell ${ }^{1}$, Faustina Hwang ${ }^{1}$, Alexis Kirke ${ }^{2}$, Asad Malik ${ }^{1}$, Etienne Roesch ${ }^{1}$, \\ James Weaver ${ }^{1}$, Duncan Williams ${ }^{2}$, Eduardo Miranda ${ }^{2}$, and Slawomir J. Nasuto ${ }^{1}$
}

\begin{abstract}
The neural mechanisms of music listening and appreciation are not yet completely understood. Based on the apparent relationship between the beats per minute (tempo) of music and the desire to move (for example feet tapping) induced while listening to that music it is hypothesised that musical tempo may evoke movement related activity in the brain.

Participants are instructed to listen, without moving, to a large range of musical pieces spanning a range of styles and tempos during an electroencephalogram (EEG) experiment. Event-related desynchronisation (ERD) in the EEG is observed to correlate significantly with the variance of the tempo of the musical stimuli. This suggests that the dynamics of the beat of the music may induce movement related brain activity in the motor cortex.

Furthermore, significant correlations are observed between EEG activity in the alpha band over the motor cortex and the bandpower of the music in the same frequency band over time. This relationship is observed to correlate with the strength of the ERD, suggesting entrainment of motor cortical activity relates to increased ERD strength.
\end{abstract}

\section{INTRODUCTION}

Music is known to have a profound set of effects on the listener. These include a wide range of emotional effects, such as changes in feelings of pleasantness, excitement etc. [1] as well as physical effects, such as changes in heart rate and desire to move [2]. However, the neural mechanisms by which music acts upon the brain to produce this apparent desire to move are not yet completely understood.

Nietzsche is quoted as saying "We listen to music with our muscles", and it has often been observed that pieces of music exert powerful influences over the body [3]. This influence of music on the body may be observed to relate to the tempo (or beat) of the music. Specifically, this applies to entrained movements such as tapping, and other movements, which are often synchronised with the beat of the music [4], [5].

This entrainment of movement to music tempo is only observed in humans and a few other species [2]. In [6] 9 participants listened to 5 rhythmic auditory stimuli and were instructed to tap along with their hands. Steady state evoked potentials were elicited in electroencephalogram (EEG) frequencies corresponding to the tempo of the auditory stimuli over the frontal cortex. This result is analogous to steady state

*This work was supported by the EPSRC grants (EP/J003077/1 and $\mathrm{EP} / \mathrm{J} 002135 / 1)$.

${ }^{1}$ I. Daly, J. Hallowell, F. Hwang, A. Malik, E. Roesch, J. Weaver, and S.J. Nasuto are with the Brain Embodiment Lab, School of Systems Engineering, University of Reading, Reading, RG6 6AY, UK i.daly at reading.ac.uk

${ }^{2}$ A. Kirke, D. Williams, and E. Miranda are with the Interdisciplinary Centre for Computer Music Research, University of Plymouth, Plymouth, PL4 8AA, UK visual evoked potentials (SSVEPs) produced by rhythmic visual stimuli [7]. However, further exploration is required to determine if similar effects are also induced by more complex and realistic musical stimuli.

To this end, we use a large set of musical stimuli extracted from film scores [8]. This allows us to make an exploration of the process by which musical tempo entrains movement and the related brain activity across a range of musical styles.

We suggest that, even when no overt movement is performed, the tempo of the music may be entraining movement related activity in the brain. Therefore, we make the hypotheses that (1) when listening to music with a particular tempo, neural correlates of movement will be observed in the brain and (2) that these neural correlates will relate to the tempo of the music being played.

Subsequently, we set out to explore the relationship between the tempo of music and whether any neural correlates of movement are observed.

The most well studied neural correlate of movement is the event-related (de)synchronisation (ERD/S). This refers to a significant reduction (desychronisation) or increase (synchronisation) from baseline in alpha $(8-13 \mathrm{~Hz})$ and/or beta $(13-30 \mathrm{~Hz})$ frequency band activity in the EEG recorded over the motor cortex that may be observed to occur before and during movement execution and imagery [9]. ERD/S strength has also been shown to correspond to a number of other cognitive processes such as hand mental rotation [10].

Thus, our hypotheses may be restated as, firstly, an ERD/S will be observed during music listening tasks, and secondly, the strength of the ERD/S will be significantly related to the tempo of the music the participant is listening to.

\section{METHODS}

\section{A. Participants}

Thirty one individuals voluntarily participated in the study. Each participant gave informed consent as per the procedures of the University of Reading research ethics committee. The participants had a median age of 35 (range 18 - 66, standard deviation 14.46). Eighteen of the participants were female and 2 were left-handed.

\section{B. EEG recording}

EEG was recorded via a Brain Products BrainAmp amplifier (Brain Products, Germany) at a sample rate of 1,000 Hz. Electrodes were positioned according to the international 10/20 system at positions Fp1, Fp2, F7, F3, Fz, F4, F8, T3, C3, Cz, C4, T4, T5, P3, Pz, P4, T6, O1, and O2. The reference channel was placed at $\mathrm{FCz}$ and the ground at $\mathrm{AFz}$. 
Impedances for all electrodes were below $5 \mathrm{k} \Omega$ for 24 of the participants and below $15 \mathrm{k} \Omega$ for all the participants.

\section{Musical stimuli}

A set of 110 excerpts from film scores were used as musical stimuli [8]. These film clips were originally selected to produce a range of different emotional responses in the listener. However, they also span a range of styles and contain a range of different tempos. Therefore, they are repurposed for our current study into the effects of tempo on the EEG.

\section{Paradigm}

The paradigm involved 6 runs of EEG recording. The first and last runs were resting state runs during which participants were instructed to sit still and rest for $300 \mathrm{~s}$. The other 4 runs each contained 10 music listening trials.

In each trial a fixation cross was presented on-screen from $t=-3 \mathrm{~s}$ (relative to the start of the music) until $t=12 \mathrm{~s}$. From $t=0 \mathrm{~s}$ the music was played until $t=12 \mathrm{~s}$ and participants were instructed to listen without moving or blinking. After $t=12 \mathrm{~s}$ the participants were asked a series of questions intended to assess their emotional responses to the music (the results of this question section are presented elsewhere). Trial timing is illustrated in figure 1.

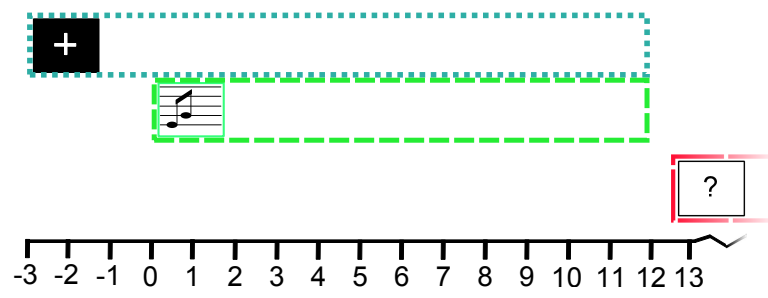

Fig. 1. Timing of the paradigm during a music listening trial. Times are reported in seconds relative to the start of the music.

\section{E. Acoustic features}

Beats per minute may be used to describe the tempo of a piece of music. However, orchestral pieces (such as the film clips used as our stimuli), in which multiple instruments are played simultaneously, may make it hard to accurately describe perceived tempo from the original score.

Therefore, a signal processing approach is taken to identify the tempo of our stimuli. Beat tracking of the music clips is performed via the dynamic programming approach proposed in [11]. The mean and standard deviation of the beats per minute throughout each music clip were estimated to allow estimation of EEG correlates corresponding to both the mean tempo and the dynamics (standard deviation) of the tempo.

\section{F. Event-related (de)synchronisation}

We estimate ERD/S strength as the mean alpha band power change on each of the EEG channels during the music listening task relative to the mean band power during the fixation cross display time in our paradigm $(t=-3 \mathrm{~s}$ to $t=0 \mathrm{~s})$. Mean ERD is calculated in a sliding window of length $1,000 \mathrm{~ms}$, step-size $100 \mathrm{~ms}$.

\section{G. Analysis}

The EEG was visually inspected for artefacts by a scorer blinded to the contents of the trials, and time segments containing artefacts were labelled as such. Independent component analysis (ICA) was then used to attempt to remove sources of artefact noise from the data. The Infomax implementation of the ICA algorithm [12] was chosen for this step. ICs were projected to the scalp one at a time and visually inspected for artefacts. ICS which were visually judged to contain artefacts were removed. Finally, any trials containing muscle or failing electrodes artefacts or amplitudes greater than $\pm 100 \mu \mathrm{V}$ (the accepted amplitude range of "clean" EEG [13]) were removed. Further details are available in [14].

Trials were first investigated to determine if an ERD/S was present in the EEG while participants listened to music. Detection of significant ERD/S effects was performed via the method described in [15].

A 10x10 cross-fold train and validation scheme was then used to identify which channels and time segments exhibited maximum correlation between ERD/S strength and either the mean or the standard deviation of the music tempo. Within each fold an exhaustive search was made of the training set to identify the time segment and channel at which ERD/S strength maximally correlated to music tempo. This channel and time segment was then tested on the held out set.

Finally, entrainment effects were investigated. Entrainment refers to induction of oscillations in a system $A$ by repeated, systematic stimulation from another system $B$. Thus, for music to be entraining movement or the desire to move there must be a regular stimulation of EEG via the moment to moment chan ges in amplitude of the music. This will be manifested in a measurable relationship over time between music and EEG. We suggest that the apparent entrainment of movement to music is due to some temporal relationship between brain activity in the motor cortex and changes in some property of the music over time.

Therefore, we investigate if there are any significant correlations between EEG in the alpha band recorded over the motor cortex and music filtered with the same frequency passband. The time series of music amplitude values was downsampled to a common sampling rate with the EEG $(100 \mathrm{~Hz})$. Alpha bandpower over time was then calculated from both the EEG and the music as the root mean squared amplitude of the signals filtered with the passband $8-13 \mathrm{~Hz}$.

Finally, entrainment hypothesises that if there is a relationship between EEG and music this will induce movementrelated brain activity. Thus, the relationship strength between music and EEG (correlation) is hypothesised to correlate with ERD/S strength at the channel and time range identified via the cross-fold validation method.

\section{RESULTS}

After artefact removal 360 trials were discounted from the analysis. Additionally, because of the known relationship between ERD strength and handedness [16] the two lefthanded participants were also excluded from this analysis. 
Thus, $31.03 \%$ of trials were removed due to artefacts and a total of 804 trials were left for analysis.

Significant ERD/S effects were observed during music listening tasks. These are illustrated in figure 2. Note that ERD effects are apparent at a range of frequencies from approximately $2 \mathrm{~s}$ after the start of the music. These are most prominent in the alpha and upper beta $(20-30 \mathrm{~Hz})$ frequency bands, particurly on channel C3.

After application of the $10 \times 10$ cross-fold validation significant correlations are found to be greatest between the variance of the tempo and the ERD/S magnitude on channel $\mathrm{C} 3$ at times $2-7 \mathrm{~s}$. The correlation peaks $4 \mathrm{~s}$ after the music begins $(r=0.143, p<0.001)$. This is illustrated in figure 3 .

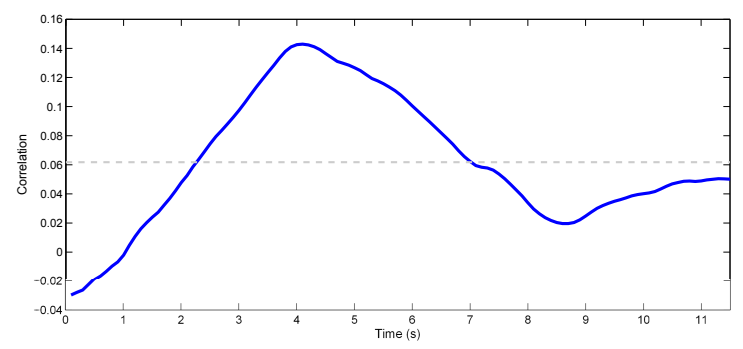

Fig. 3. Correlation over time between ERD/S magnitude and tempo variance in the music on channel C3. The dotted line indicates statistical significance $(p<0.05$, corrected for multiple comparisons via Bonferroni).

Figure 4 illustrates the scalp map of correlations between ERD/S strength and the variance of the tempo of the music at time segment $2-7 \mathrm{~s}$. Note that the strongest correlations are found over the left motor cortex, which is also the location of the greatest ERD/S effects.

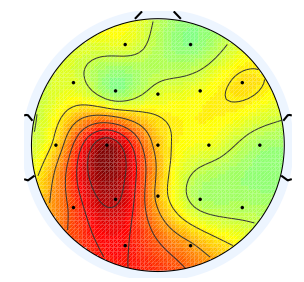

Fig. 4. Scalp map of correlations between tempo of the musical stimuli and ERD/S strength at time segment 2-7 s for each channel.

Statistically significant correlations are found between the music and the EEG in the alpha frequency band in the range $r=-0.693$ to $r=0.764$ (median: $r=-0.042$ ). This relationship correlates significantly with the ERD/S strength recorded on channel $\mathrm{C} 3$ at the time segment of interest $(2-7 \mathrm{~s})$ $(r=-0.162, p<0.001)$.

It may be suggested that the observed ERD effects are a result of an auditory steady-state response (ASSR) [17]. An ASSR is evoked to by a repeated audio stimulus at a fixed frequency (for example, an amplitude modulated tone at $10 \mathrm{~Hz}$ ). Thus, to determine if the observed effects are due to an ASSR evoked by such a fixed stimulus partial correlation is used to identify the correlation between EEG in the alpha band and the music filtered into the same passband when sine waves at frequencies of $8-13 \mathrm{~Hz}$ are also considered.
No significant difference is observed between the correlation of EEG with music in the alpha band and the partial correlation of EEG with music and additional sine waves. Thus, the observed relationships between music, EEG, and ERD strength are not simply the result of ASSRs induced by a fixed frequency stimulus but rather motor related activity induced by music tempo.

\section{DISCUSSION}

The variance of the tempo of the music played to our participants is found to correlate significantly with the strength of the event-related desynchronisation (ERD) recorded over channel C3 over the left motor cortex. Thus, the more variable the tempo in the music played to the participants the stronger their ERD response.

All participants were instructed to sit still throughout the music listening task. Thus, ERD related effects are not due to cued or spontaneous movement of the participants.

It is interesting that ERD in the left motor cortex is most significantly correlated with music tempo change. All participants in this study were right-handed, thus, the tempo of the music on the contra-lateral hemisphere to the dominant hand is found to be most strongly correlated to tempo.

It may be argued that the observed neural correlates of musical tempo are a form of auditory steady state response (ASSR) (as reported in [6]). However, we think this is unlikely as ASSRs occur symmetrically over the pre-frontal cortex, while the effects we observe occur over the motor cortex confined to the area contra-lateral to the dominant hand. Furthermore, they are confined to the alpha frequency band, which is known to be associated with movement [9].

It is interesting to note that no significant correlation with the mean tempo is found. Thus, ERD/S strength may be said to be modulated by the variation (dynamics) of the musical tempo, but a piece of music with a steady tempo (either fast or slow) over the duration of the music presentation time does not significantly modulate ERD/S strength. ASSRs are induced by steady stimuli with little or no variance. As the observed ERD/S strength is not modulated by a constant stimuli (as shown by the lack of significant difference between correlation of music with EEG and partial correlation with additional sine waves between $8-13 \mathrm{~Hz}$ considered) this provides further evidence that the observed brain activations are motor related and not ASSRs.

It has been shown elsewhere that music tonality can produce significant changes in the motor cortex in an fMRI study [18]. Thus, our findings of tempo induced motor related ERD/S activity may be somewhat related. Furthermore, the ERD/S effects may be due to an expectation / surprise effect related to the music tempo. The greater the variation of the tempo the greater the level of surprise that may be induced. In [19] alpha bandpower on right central channels is reported to provide high classification for identifying music stimuli selected to provide "surprise". Additionally, in [20] ERD is reported to relate to anticipation of an auditory stimulus.

A large number of our trials exhibit significant correlations between alpha bandpower recorded over the motor cortex and 

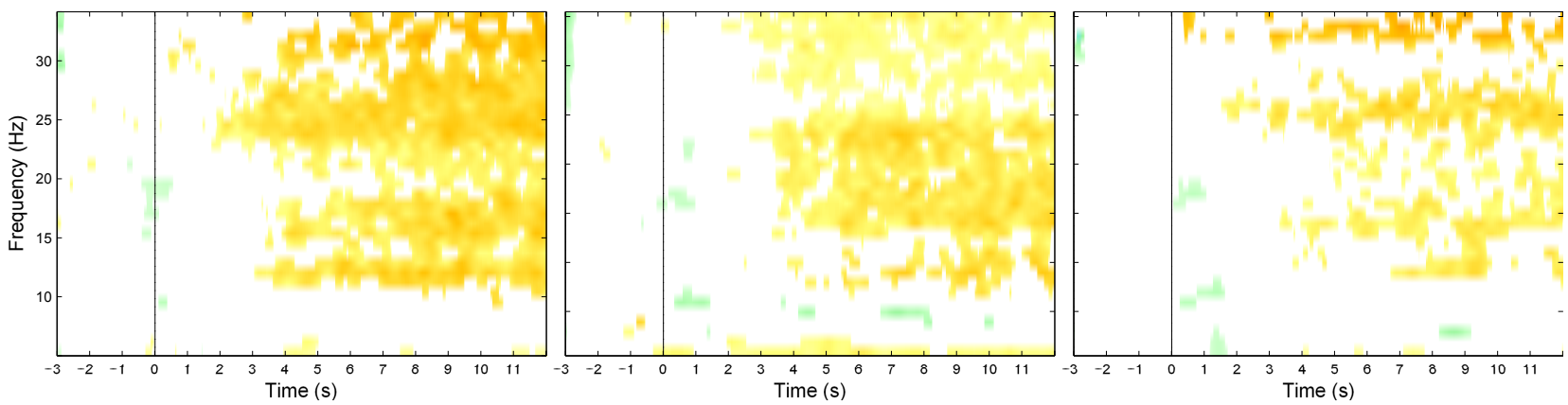

Fig. 2. ERD/S observed on channels $\mathrm{C} 3$ (left), $\mathrm{Cz}$ (middle), and $\mathrm{C} 4$ (right) during music listening tasks. The redder (orange) colours indicate significant ERD and the bluer (light green) colours significant ERS $(p<0.01)$. Significance is tested via the method described in [15].

corresponding bandpower in the music. This suggests that the music is entraining activity in the motor cortex at this frequency band. The correlation between this relationship and the ERD strength further suggests that this entrainment is modulating motor cortex activation. Although entrainment is a non-linear phenomenon, it may account for the apparently linear changes in ERD, which have been demonstrated to arise from interactions of a wide range of both linear and non-linear coupled oscillators [21].

Our future work will seek to provide further understanding of the neural processes that are involved in music listening. It is also interesting to relate musical features, such as tempo, with affective states induced by the music and, thus, arrive at a neural mechanism by which combinations of musical features produce particular sets of emotions.

Our findings may also have impact in the construction of brain-computer music interfaces (BCMIs), devices for interacting with music via brain activity [22]. For example, $\mathrm{ERD} / \mathrm{S}$ is a common control mechanism in $\mathrm{BCI}$ and it is reasonable to speculate that it may also be used to allow BCMI users to control aspects of music such as tempo.

\section{ACKNOWLEDGMENTS}

This work was supported by the EPSRC grants (EP/J003077/1 and EP/J002135/1). The authors thank Sajeel Ahmed, Isil Poyraz Bilgin, Katherine Heseltine Flynn, and Maryam Karimidomal for help with EEG recording.

\section{REFERENCES}

[1] P. N. Juslin and D. Västfjäll, "Emotional responses to music: the need to consider underlying mechanisms.," The Behavioral and brain sciences, vol. 31, pp. 559-75; discussion 575-621, Oct. 2008.

[2] A. Schachner, T. F. Brady, I. M. Pepperberg, and M. D. Hauser, "Spontaneous motor entrainment to music in multiple vocal mimicking species.," Current biology : CB, vol. 19, pp. 831-6, May 2009.

[3] O. Sacks, "The power of music.," Brain : a journal of neurology, vol. 129, pp. 2528-32, Oct. 2006.

[4] J. Phillips-Silver, C. A. Aktipis, and G. A. Bryant, "The ecology of entrainment: Foundations of coordinated rhythmic movement.," Music perception, vol. 28, pp. 3-14, Sept. 2010.

[5] M. Leman, D. Moelants, M. Varewyck, F. Styns, L. van Noorden, and J.-P. Martens, "Activating and relaxing music entrains the speed of beat synchronized walking.," PloS one, vol. 8, p. e67932, Jan. 2013.

[6] S. Nozaradan, I. Peretz, and A. Mouraux, "Selective neuronal entrainment to the beat and meter embedded in a musical rhythm.," The Journal of neuroscience : the official journal of the Society for Neuroscience, vol. 32, pp. 17572-81, Dec. 2012.
[7] F. Di Russo, S. Pitzalis, T. Aprile, G. Spitoni, F. Patria, A. Stella, D. Spinelli, and S. A. Hillyard, "Spatiotemporal analysis of the cortical sources of the steady-state visual evoked potential.," Human brain mapping, vol. 28, pp. 323-34, Apr. 2007.

[8] T. Eerola and J. K. Vuoskoski, "A comparison of the discrete and dimensional models of emotion in music," Psychology of Music, vol. 39, pp. 18-49, Aug. 2010.

[9] G. Pfurtscheller and F. Lopes da Silva, "Event-related EEG/MEG synchronization and desynchronization: basic principles," Clinical Neurophysiology, vol. 110, pp. 1842-1857, Nov. 1999.

[10] X. Chen, G. Bin, I. Daly, and X. Gao, "Event-related desynchronization (ERD) in the alpha band during a hand mental rotation task," Neuroscience Letters, Feb. 2013.

[11] D. P. W. Ellis, "Beat Tracking by Dynamic Programming," Journal of New Music Research, vol. 36, pp. 51-60, Mar. 2007.

[12] A. J. Bell and T. J. Sejnowski, "An information-maximization approach to blind separation and blind deconvolution.," Neural computation, vol. 7, pp. 1129-59, Nov. 1995.

[13] I. Daly, F. Pichiorri, J. Faller, V. Kaiser, A. Kreilinger, R. Scherer, and G. Mueller-Putz, "What does clean EEG look like?," in Conf Proc IEEE Eng Med Biol Soc, 2012.

[14] I. Daly, A. Malik, F. Hwang, E. Roesch, J. Weaver, A. Kirke, D. Williams, E. Miranda, and S. J. Nasuto, "Neural correlates of emotional responses to music: an EEG study," Neuroscience Letters, vol. 573, pp. 52-57, May 2014.

[15] B. Graimann, J. E. Huggins, S. P. Levine, and G. Pfurtscheller, "Visualization of significant ERD/ERS patterns in multichannel EEG and ECoG data.," J Clin Neurophysiol, vol. 113, pp. 43-7, Jan. 2002.

[16] A. Stancák and G. Pfurtscheller, "The effects of handedness and type of movement on the contralateral preponderance of $\mu$-rhythm desynchronisation," Electroencephalography and Clinical Neurophysiology, vol. 99, no. 2, pp. 174-182, 1996.

[17] D. W. F. Schwarz and P. Taylor, "Human auditory steady state responses to binaural and monaural beats.," Clinical neurophysiology : official journal of the International Federation of Clinical Neurophysiology, vol. 116, pp. 658-68, Mar. 2005.

[18] S. Durrant, E. R. Miranda, D. R. Hardoon, J. Shawe-taylor, A. Brechmann, and H. Scheich, "Neural Correlates of Tonality in Music," in Proceedings of Music, Brain \& Cognition Workshop - NIPS Conference, Whistler (Canada), 2007.

[19] R. Khosrowabadi, A. Wahab, K. K. Ang, and M. H. Baniasad, "Affective computation on EEG correlates of emotion from musical and vocal stimuli," in 2009 International Joint Conference on Neural Networks, pp. 1590-1594, IEEE, June 2009.

[20] M. C. Bastiaansen, K. B. Böcker, P. J. Cluitmans, and C. H. Brunia, "Event-related desynchronization related to the anticipation of a stimulus providing knowledge of results.," Clinical neurophysiology: official journal of the International Federation of Clinical Neurophysiology, vol. 110, pp. 250-60, Feb. 1999.

[21] J. H. Sheeba, V. K. Chandrasekar, and M. Lakshmanan, "General coupled-nonlinear-oscillator model for event-related (de)synchronization," Physical Review E, vol. 84, p. 036210, Sept. 2011.

[22] E. R. Miranda, "Brain-Computer music interface for composition and performance," IntJ Dis Human Dev, vol. 5, no. 2, 2006. 